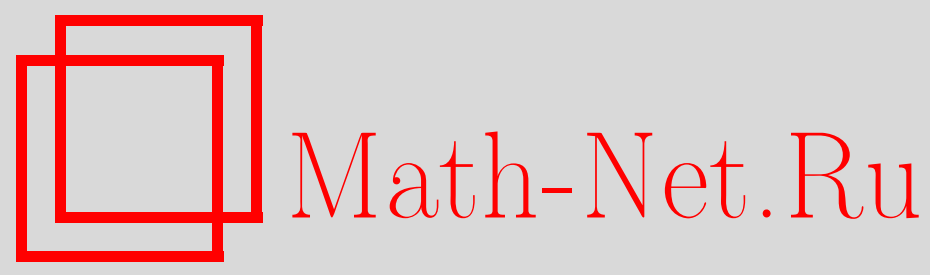

М. М. Гриненко, О расслоениях на поверхности дель Пеццо, Матем. заметки, 2001, том 69, выпуск 4, 550-565

DOI: https://doi.org/10.4213/mzm522

Использование Общероссийского математического портала Math-Net.Ru подразумевает, что вы прочитали и согласны с пользовательским соглашением http://www.mathnet.ru/rus/agreement

Параметры загрузки:

IP : 3.89 .185 .249

26 апреля 2023 г., 13:39:56

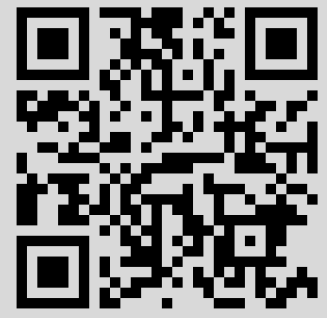




\title{
О РАССЛОЕНИЯХ НА ПОВЕРХНОСТИ ДЕЛЬ ПЕЦЦО
}

\author{
М.М. Гриненко
}

\begin{abstract}
В данной заметке обсуждается проблема бирациональной жесткости для трехмерных алгебраических многообразий, расслоенных над рациональной кривой на поверхности дель Пеццо степени 1. Предлагается критерий жесткости таких расслоений в категории Мори и приводится доказательство обратной импликации (теорема 3.3). Рассматривается также вопрос о перестройках слоев данного типа расслоений, который оказывается тесно связан с проблемой жесткости. В частности, формулируется важный результат о единственности гладкой модели в классе отображений над базой (следствие 4.5).
\end{abstract}

Библиограф̆ия: 20 названий.

1. Введение. Проблема бирациональной классификации алгебраических многообразий большой (3 и вьше) размерности, несмотря на то, что это всего лишь часть общей задачи классификации, вплоть до настоящего времени остается в числе труднейших. Если этап такой классификации, основанный на кодаировой размерности, не вызывает сомнений, то ответ на вопрос "а что дальше?" до сих пор неочевиден. Опыт последних двадцати лет показывает (см., например, [1]-[4]), что для многообразий отрицательной кодаировой размерности следующим этапом может быть деление на "жесткие" и "нежесткие".

Предположим, что $M$ - гладкое трехмерное алгебраическое многообразие с $k(M)=$ $-\infty$ над алгебраически замкнутым полем нулевой характеристики. Применяя к $M$ программу минимальных моделей (хороший обзор этой программы содержится в [5]), мы получаем бирациональное отображение $\iota: M \rightarrow X$ на некоторое Мори-расслоение $X$. Напомним, что алгебраическое многообразие $X$ называется расслоением в смысле $M о$ $p u$ (или, короче, Мори-расслоением), если вьполнены следуюшие условия:

1) $X$ имеет $\mathbb{Q}$-факториальные терминальные особенности;

2) существует экстремальное стягивание $\pi: X \rightarrow S$ расслоенного типа (в частности, $S$ - нормальное алгебраическоемногообразие строго меньшей размерности, антиканонический дивизор $-K_{X}$ относительно обилен и $\left.\rho(X / S)=1\right)$.

Отметим, что $X$ и ८ редко бывают определены однозначно: например, $X$ может иметь бирациональные автоморфизмы или перестройки на другие Мори-расслоения. Также заметим, что Мори-расслоения составляют более-менее обозримьй класс многообразий, так что вполне резонна следующая переформулировка задачи бирациональной

Работа выполнена при поддержке гранта Российского фонда фундаментальных исследований № 99-01-01132, гранта поддержки ведущих научных школ № 00-15-96085, гранта NWO-RFBR № 047-008-005 и гранта INTAS-OPEN № 97/2072. 
классификации: в данном классе бирациональной эквивалентности описать все модели Мори-расслоений и перестройки (т.е. бирациональные отображения) между ними.

Экстремальное стягивание расслоенного типа в определении расслоения в смысле Мори играет ключевую роль в делении многообразий на жесткие и нежесткие. Пусть $\pi: X \rightarrow S$ и $\pi^{\prime}: X^{\prime} \rightarrow S^{\prime}$ - некоторые Мори-расслоения, бирациональные друг другу посредством отображения $\chi$. Тогда указанное деление определяется, грубо говоря, ответом на вопрос, индуцирует ли $\chi$ бирациональное отображение между базами $S$ и $S^{\prime}$ наших Мори-расслоений. Точное определение зависит от размерности базы расслоения:

ОПРЕДЕЛЕНИЕ 1.1. Мори-расслоение $\pi: X \rightarrow S, \operatorname{dim} S>0$, называется (бирационально) жестким, если любое бирациональное отображение $\chi: X \rightarrow X^{\prime}$ на другое Мори-расслоение $\pi^{\prime}: X^{\prime} \rightarrow S^{\prime}$ проводится через базу, т.е. существует бирациональное отображение $\mu: S \rightarrow S^{\prime}$, замыкающее следующую диаграмму до коммутативной:

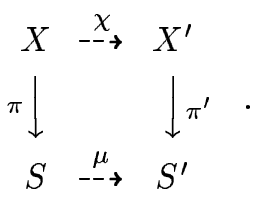

$\mathbb{Q}$ - ано многообразие $X$ (т.е. Мори-расслоение $\pi: X \rightarrow S$ c $\operatorname{dim} S=0$ ) называется жестким, если любое Мори-расслоение, бирациональное $X$, бирегулярно изоморфно $X$.

Очевидно, свойство “быть жестким” бирационально инвариантно. По сути дела, это свойство означает единственность модели Мори-расслоения с точностью до бирациональных отображений над базой.

Для трехмерных Мори-расслоений $X / S$ сложилась определенная терминология (в

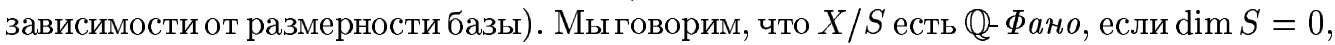
расслоение на коники, если $\operatorname{dim} S=2$, и, наконец, расслоение на поверхности дель Пецио степени $d$, если $\operatorname{dim} S=1$ и его слой $F_{\eta}$ над общей схемной точкой $S_{\eta}$ имеет степень $d=\left(K_{F_{\eta}}\right)^{2}$. Отметим, что в последнем случае $F_{\eta}$ есть гладкая поверхность дель Пеццо над незамкнутым полем (т.е. над полем функций кривой $S$ ) с числом Пикара, равным единице.

Вот некоторые примеры жестких и нежестких многообразий.

1. $\mathbb{Q}$-Фано. Самый известньй пример жесткого трехмерного многообразия Фано гладкая квартика в $\mathbb{P}^{4}$. В работе [6] доказано, что любое ее бирациональное отображение на Мори-расслоение является бирегулярньм изоморфизмом.

Среди нежестких наиболее известна гладкая кубика в $\mathbb{P}^{3}$ : она перестраивается и в

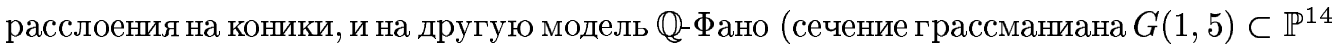
подпространством коразмерности 5).

2. Расслоения на коники. Пусть $X \rightarrow \mathbb{P}^{2}$ - стандартное расслоение на коники с кривой вырождения $D$. Тогда если $\operatorname{deg} D \geqslant 12$, то $X / \mathbb{P}^{2}$ бирационально жестко. Работа [3], где это доказано, послужила отправной точкой для создания программы Саркисова.

Примером нежесткого может быть та же кубика в $\mathbb{P}^{4}$ : она перестраивается в стандартное расслоение на коники с $\operatorname{deg} D=5$. 
3. Расслоения на поверхности дель Пеццо. Простейший пример жесткого расслоения на поверхности дель Пеццо степени 2 над рациональной кривой может быть построен следуюшим образом: надо взять двойное накрытие $\mathbb{P}^{1} \times \mathbb{P}^{2}$ с ветвлением в дивизоре би-степени $(2,4)$. Многочисленные примеры даны в [2] и [7].

Первьй (полностью разобранньй с точки зрения проблемы жесткости) пример нежесткого расслоения на поверхности дель Пеццо приведен в [8]. Это так назьваемый двойной конус, т.е. двойное накрытие невырожденного квадратичного конуса в $\mathbb{P}^{4} \mathrm{c}$ ветвлением в гладком сечении квартикой. Прообразы двух семейств плоскостей конуса образуют два пучка поверхностей дель Пеццо степени 2, которые дают структуру Мори-расслоения в зависимости от того, какое из двух возможных малых разрешений особенностей двойного конуса мы выбираем.

В данной статье мы будем рассматривать только трехмерные Мори-расслоения на поверхности дель Пещцо $X / S$ степени 1. Но прежде сделаем два общих замечания о проблеме жесткости для расслоений на поверхности дель Пещцо произвольной степени.

Во-первых, всегда можно полагать $S$ рациональной кривой. Действительно, если род $p_{g}(S)>0$, то проблема жесткости $X / S$ легко решается из того соображения, что общий слой покрьвается рациональными кривыми, и полный ответ достигается применением теоремы 2.6 работы [9].

Во-вторьх, степень $d$ общего слоя $X / \mathbb{P}^{1}$ должна быть не более 3 . В самом деле, если $d \geqslant 5$, то $F_{\eta}$ рационально над полем функций $\mathbb{P}^{1}[10]$, поэтому $X$ само рационально и, очевидно, всегда нежестко. Если же $d=4$, то это также нежесткий случай: раздутие (общего) сечения $X / S$ дает расслоение на коники.

Большая часть данной статьи была написана автором во время визита в Математический институт Макса Планка в Бонне. Пользуясь удобньм случаем, автор выражает свою искреннюю признательность руководству и персоналу института за приглашение и комфортные условия для работы.

2. Вспомогательный материал. В этом разделе кратко описан технический инструмент, используемый в статье. Все необходимые понятия из программы минимальных моделей читатель может найти в [11] и [5].

Пусть $\pi: V \rightarrow S$ и $\pi^{\prime}: V^{\prime} \rightarrow S^{\prime}$ - трехмерные Мори-расслоения, $\chi: V \rightarrow V^{\prime}-$ бирациональное отображение, $\mathscr{H}^{\prime}=\left|n^{\prime}\left(-K_{V^{\prime}}\right)+\pi^{\prime *}\left(A^{\prime}\right)\right|-$ непустая линейная система без базисных точек, $A^{\prime}$ - обильный дивизор на $S^{\prime}$ и $\mathscr{H}=\chi_{*}^{-1} \mathscr{H}^{\prime} \subset\left|n\left(-K_{V}\right)+\pi^{*}(A)\right|-$ собственньй прообраз $\mathscr{H}^{\prime}$ на $V$, где $A$ - некоторьй дивизор Вейля на $S$. Верны следующие утверждения [12, предложение 3.5 и теорема 4.2$]$

ПРЕДЛОЖЕНИЕ 2.1. 1. Всегда $n \geqslant n^{\prime}$ и в случае равенства отображсние $\chi$ бирачионально над базой (т.е. индуцирует бирациональное отображение $S \rightarrow S^{\prime}$, согласованное со структурой расслоений).

2. Если система $\mathscr{H}^{\prime}$ обильна и лог-пара $K_{V}+\frac{1}{n} \mathscr{H}$ канонична и численно эффективна, то ұ - бирегулярный изоморфизм над базой.

3. Если отображсение ұ бирачионально над базой и является изоморфизмом в коразмерности 1, то этот изоморфизм бирегулярен.

Напомним основные понятия программы Саркисова. Каждый шаг этой программы стартует с некоторого Мори-расслоения $X / S$ и дает другое Мори-расслоение $X^{\prime} / S^{\prime}$ при помощи одной из четырех әлементарных перестроек, описанных ниже (в англоязычной литературе используются слова "elementary links"). Цель программы состоит в 
том, чтобы данное бирациональное отображение $\chi: V \rightarrow V^{\prime}$ между Мори-расслоениями $V / S$ и $V^{\prime} / S^{\prime}$ разложить в композицию таких элементарных перестроек. В следующих диаграммах все стягивания экстремальны по отношению к некоторым лог-парам, все пунктирные стрелки обозначают последовательность лог-флипов. Итак, опишем эти четыре типа элементарных перестроек.

ЭЛЕМЕНТАРНАЯ ПЕРЕСТРОЙКА ТИПА 1. Эта перестройка задается диаграммой

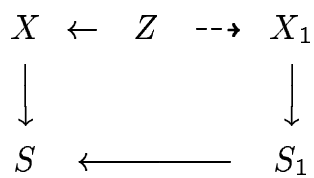

Здесь $Z \rightarrow X$ - дивизориальное стягивание, $S_{1} \rightarrow S$ - морфизм со связньгии слоями, $\rho\left(S_{1} / S\right)=1$.

ЭЛЕМЕНТАРНАЯ ПЕРЕСТРОЙКА ТИПА 2 задается диаграммой

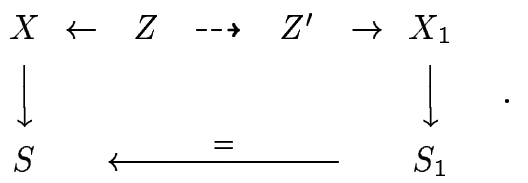

Здесь $Z \rightarrow X$ и $Z^{\prime} \rightarrow X^{\prime}$ - дивизориальные стягивания.

ЭЛЕМЕНТАРНАЯ ПЕРЕСТРОЙКА ТИПА 3 задается диаграммой

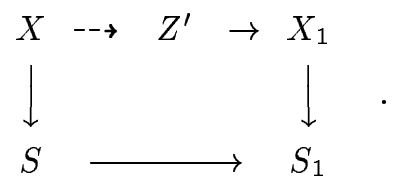

Это - обращение элементарной перестройки типа 1.

ЭЛЕМЕНТАРНАЯ ПЕРЕСТРОЙКА ТИПА 4 задается диаграммой

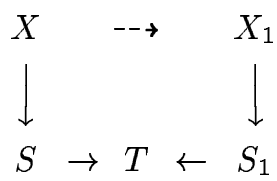

Здесь $S \rightarrow T$ и $S_{1} \rightarrow T-$ морфизмы со связными слоями, $T$ нормально и $\rho(S / T)=$ $\rho\left(S_{1} / T\right)=1$.

Известно [13], [12], что процедура разложения существует и обрьвается через конечное число шагов.

Следующий важньй факт доказан в [9, теорема 2.6].

ПРЕДЛОЖЕНИЕ 2.2. Пусть $K$ - совершенное (алгебраически незамкнутое) поле, $X / S$ и $X_{1} / S_{1}$ - двумерные Мори-расслоения, определенные над $K$ и бирационально изоморфные посредством отобрахения $\chi, S=\operatorname{Spec} K u X-$ поверхность дель Пецио степени 1 c $\operatorname{Pic}(X) \sim \mathbb{Z}$. Тогда $X \cong X_{1}$, а отображсение $\chi$ бирегулярно.

Наконец, нам потребуется ряд фактов о горенштейновых поверхностях дель Пецо степени 1. Доказательства приводимьх ниже результатов можно найти в [14, предложение 4.6] и [15, следствие 4.10]. 
ПРЕДЛОЖЕНИЕ 2.3. Пусть $F$ - приведенная неприводимая горенитейновая поверхность дель Пецио степени 1, являющаяся схемой Коэна-Маколея и определенная над алгебраически замкнутым полем нулевой характеристики. Тогда антиканоническая линейная система не имеет базисных точек и дает морфизм степени 2 на квадратичный конус в $\mathbb{P}^{3}$ с ветвлением вдоль сечения кубикой, не проходящей через вериину конуса; единственная базисная точка $\left|-K_{F}\right|$ лежит над этой верииной.

3. Условие жесткости. Пусть $\pi: V \rightarrow \mathbb{P}^{1}$ - трехмерное Мори-расслоение на поверхности дель Пеццо степени 1 . Тогда группа классов $\mathbb{Q}$-дивизоров Вейля порождена классами антиканонического дивизора $-K_{V}$ и слоя $F$, т.е.

$$
\mathrm{Wl}_{\mathbb{Q}}(V)=\mathbb{Q}\left[-K_{V}\right] \oplus \mathbb{Q}[F]
$$

где $F=\pi^{*}$ (pt.). Далее, если $\mathscr{D}$ - непустая линейная система, то $\mathscr{D} \subset\left|n\left(-K_{V}\right)+m F\right|$ для некоторого неотрицательного числа $n$, которое назьвается квазиәффективны.м порогом и обозначается $\mu(V, \mathscr{D})$.

Число $m$ определяет "закрутку” по базе (относительно $n\left(-K_{V}\right)$ ) дивизоров линейной системы и, вероятно, играет решающую роль в проблеме жесткости. А именно, имеет место

ГИПотеЗА 3.1. Пусть $V / \mathbb{P}^{1}$ - Мори-расслоение на поверхности дель Пецио степени 1. Тогда $V / \mathbb{P}^{1}$ бирачионально жестко, если и только если для любого $n>0$ линейная система $\left|n\left(-K_{V}\right)-F\right|$ либо пуста, либо имеет неподвижную компоненту.

ЗАМЕЧАНИЕ 3.2. Основой для выдвижения гипотезы послужили работы [2], [16], а также работа автора [7], где доказана справедливость гипотезы 3.1 в гладких случаях.

Хотя мы, вероятно, пока весьма далеки от ответа на вопрос, верна ли сформулированная гипотеза (или при каких дополнительных условиях она верна), тем не менее в одну сторону (именно часть “только если”) мы можем ее доказать немедленно.

ТеОрема 3.3. Если Мори-расслоение $V / \mathbb{P}^{1}$ на поверхности дель Пеццо степени 1 бирационально жестко, то любая линейная система вида $\left|n\left(-K_{V}\right)-F\right|$ либо пуста, либо имеет неподвижную компоненту.

ДокАЗАТЕльСтво. Предположим обратное, т.е. $V / \mathbb{P}^{1}$ жестко и для $n \gg 0$ существует непустая полная линейная система $\mathscr{D}=\left|n\left(-K_{V}\right)-F\right|$ без неподвижных компонент.

Заметим, что лог-дивизор $K_{V}+\frac{1}{n} \mathscr{D}$ каноничен (или, другими словами, $\mathscr{D}$ не имеет максимальных особенностей) вдоль любой горизонтальной (накрьвающей базу) кривой. Действительно, предположив противное, мы найдем некоторую неприводимую горизонтальную кривую $B$ такую, что $\nu=\operatorname{mult}_{B} \mathscr{D}>n$, и тогда немедленно получим противоречие: индекс пересечения кривой из антиканонической линейной системы общего слоя, не лежащей в базисном множестве $\mathscr{D}$ и пересекающей $B$, с общим элементом $\mathscr{D}$ равен $n$ и не может быть меньше $\nu$.

Итак, мы можем считать, что $\mathscr{D}$ не имеет неподвижных компонент и лог-пара $K_{V}+\frac{1}{n} \mathscr{D}$ канонична вдоль любой горизонтальной кривой. Запустим программу Саркисова с 
целью сделать эту лог-пару каноничной. Мы получаем конечную цепочку элементарных перестроек типа 2 (тип 1 невозможен ввиду предложения 2.2)

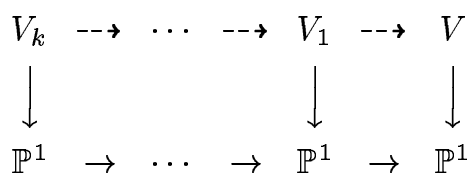

такую, что $K_{V_{k}}+\frac{1}{n} \mathscr{D}_{k}$ канонична, где $\mathscr{D}_{k}-$ собственный прообраз $\mathscr{D}$. Заметим, что в данном случае все элементарные перестройки реализованы следующим образом: вначале мы раздуваем что-то, лежащее в некотором слое, затем делаем несколько лог-флипов над базой, после чего стягиваем собственньй прообраз этого слоя. Отсюда видно, что наша цепочка перестроек дает изоморфизм над некоторым открытым подмножеством базы, поэтому $\mathscr{D}_{k} \subset\left|n\left(-K_{V_{k}}\right)-a F\right|$ (через $F$ по-прежнему обозначаем класс слоя). $\mathrm{Bce,} \mathrm{что} \mathrm{нам} \mathrm{нужно,} \mathrm{это} \mathrm{показать,} \mathrm{что} a>0$. Это следует, опять-таки, из инвариантности размерности Иитаки. Действительно, $k\left(V, K_{V}+\frac{1}{n} \mathscr{D}\right)=-\infty$, но если $a \leqslant 0$, то $k\left(V_{k}, K_{V_{k}}+\frac{1}{n} \mathscr{D}_{k}\right) \geqslant 0$ ввиду каноничности $K_{V_{k}}+\frac{1}{n} \mathscr{D}_{k}$. Итак, $a>0$ и мы можем применить элементарную перестройку типа 3 или 4 к паре $\left(V_{k}, K_{V_{k}}+\frac{1}{n} \mathscr{D}_{k}\right)$. Мы получим либо $\mathbb{Q}$-Фано (перестройка типа 3), либо расслоение на коники или поверхности дель Пеццо (перестройка типа 4). Любая из полученных структур Мори-расслоения будет отлична от $V / \mathbb{P}^{1}$ (т.е. не проводится через базу). Полученное противоречие доказывает теорему.

ЗАмЕчАниЕ 3.4. Весьма вероятно, что теорема 3.3 верна и при степени слоя, равной 2 или 3. Для ее доказательства в этих случаях достаточно показать, что непустые линейные системы вида $|n(-K)-F|$ без неподвижных компонент (если таковые существуют) при $n \gg 0$ не имеют максимальных особенностей вдоль горизонтальных кривых.

ЗАмЕчАниЕ 3.5. В случае горенштейновых расслоений на поверхности дель Пеццо степени 1 или 2 можно в явном виде предъявить бирациональную пререстройку на другое Мори-расслоение и тем самым доказать теорему 3.3. Дело в том, что мы можем воспользоваться удобными проективными моделями таких расслоений. Конструкция этих моделей извлечена автором из работы [16] и полностью, со всеми вычислениями воспроизведена в [7]. Основной факт, вытекающий оттуда, состоит в том, что из существования непустых линейных систем вида $\left|n\left(-K_{V}\right)-F\right|$ без неподвижных компонент в указанных случаях следует существование системы $\left|-K_{V}-F\right|$ с теми же свойствами и с гладким общим элементом. Если $D$ - такой элемент, то из формулы присоединения сразу следует, что $D$ - рациональная эллиптическая поверхность. А тогда мы можем выбрать общий подпучок $\mathscr{P} \subset\left|-K_{V}-F\right|$ и разрешить его базисное множество. На построенном таким образом многообразии собственный прообраз пучка задает морфизм на $\mathbb{P}^{1}$. Слои этого морфизма, очевидно, суть рациональные поверхности, и остается только применить относительный вариант программы минимальных моделей. Мы получим структуру расслоения на коники или поверхности дель Пещо, которая будет лежать “поперек” исходной. Указанная процедура, по сути дела, воспроизводит шаги программы Саркисова.

В заключение лиш отметим, что такое рассуждение непроходит для степени 3. Простейший пример дает квартика в $\mathbb{P}^{4}$, содержащая плоскость. Такое многообразие обязательно имеет не $\mathbb{Q}$-факториальные особенности (в общем случае, девять двойных точек) 
и поэтому не является Мори-расслоением. Мы, однако, можем раздуть вложенную плоскость и получить малое разрешение особенностей, а тем самым и гладкое расслоение на поверхности дель Пещо степени 3. Легко видеть, что в таком расслоении линейная система $|-3 K-F|$ непуста и не имеет неподвижных компонент, но зато $|-K-F|$ состоит из одного элемента (собственного прообраза плоскости), а все элементы $|-2 K-F|$ приводимы. Заметим, что данное малое разрешение допускает флоп, и после стягивания образа плоскости мы получим $\mathbb{Q}$ - $а$ ано с негоренштейновой точкой индекса 2.

4. Перестройки слоев. Пусть $V / \mathbb{P}^{1}$ - наше Мори-расслоение на поверхности дель Пещц степени 1. Рассмотрим три класса многообразий:

$$
\begin{aligned}
\mathfrak{B}\left(V / \mathbb{P}^{1}\right) & =\{\text { все многообразия, бирациональные } V\}, \\
\mathfrak{M}\left(V / \mathbb{P}^{1}\right) & =\{\text { Мори-расслоения, бирациональные } V\}, \\
\mathfrak{M}_{b}\left(V / \mathbb{P}^{1}\right) & =\left\{\text { Мори-расслоения, бирациональные } V / \mathbb{P}^{1} \text { над базой }\right\} .
\end{aligned}
$$

Как уже отмечалось, программа минимальных моделей перегоняет любое многообразие из $\mathfrak{B}\left(V / \mathbb{P}^{1}\right)$ в $\mathfrak{M}\left(V / \mathbb{P}^{1}\right)$. Далее, сам класс $\mathfrak{M}\left(V / \mathbb{P}^{1}\right)$ замкнут относительно программы Саркисова, другими словами, любые два его элемента связаны композицией элементарных перестроек четырех типов. Переходы внутри класса $\mathfrak{M}_{b}\left(V / \mathbb{P}^{1}\right)$, очевидно, достигаются элементарными перестройками типа 2 . Наконец, из класса $\mathfrak{M}\left(V / \mathbb{P}^{1}\right)$ в класс $\mathfrak{M}_{b}\left(V / \mathbb{P}^{1}\right)$ можно попасть только при помощи перестроек типа 4 или 1 , а обратнотипа 4 или 3 (предложение 2.2).

Легко видеть, что любая перестройка типа 2 разлагается в композицию конечного числа перестроек слоя, т.е. бирациональных отображений

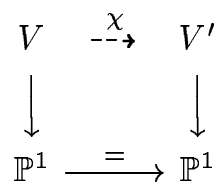

таких, что $\chi$ индуцирует изоморфизм $\chi_{\eta}: V_{\eta} \cong V_{\eta}^{\prime}$ в слое над общей схемной точкой прямой и, более того, изоморфизм всюду вне слоя над некоторой ( “обычной") точкой $\mathbb{P}^{1}$. Ясно, что для доказательства гипотезы 3.1 достаточно убедиться в сохранении условия "не иметь непустых линейных систем вида $|n(-K)-F|$ без неподвижных компонент" при перестройках слоя.

Для расслоений на поверхности дель Пещц малых степеней такие перестройки почти не рассматривались в математической литературе (единственные известные автору работы на эту тему - [17] и недавно появившийся препринт [18]). Ограничившись горенштейновыми многообразиями, мы попытаемся немного прояснить этот вопрос при степени слоя, равной 1.

Всюду далее, если не оговорено противное, мы находимся в следующих условиях. Пусть $\mathscr{O}$ - кольцо дискретного нормирования (например, локальное кольцо точки на кривой) с алгебраически замкнутым полем вычетов $k$ характеристики $0, R=\operatorname{Spec~} \mathscr{O}$, $K$ - поле функций $\mathscr{O}, X$ и $V$ - поверхности дель Пещц степени 1 над $R$, которые (как трехмерные многообразия над $k$ ) предполагаются горенштейновыми Мори-расслоениями над $R$. В частности, группы Пикара слоев $X_{K}$ и $V_{K}$ над общей схемной точкой $R$ порождены антиканоническими дивизорами. Центральные слои обозначим $X_{0}$ и $V_{0}$ соответственно, и пусть

$$
\varphi: X \rightarrow V
$$


будет бирациональным над $R$ отображением, индуцируюшим изоморфизм

$$
\varphi_{K}: X_{K} \simeq V_{K}
$$

ЗАмЕчАниЕ 4.1. В недавнем препринте [18] показано, что вплоть до степени слоя, равной 4 , отображение $\varphi$ всегда является изоморфизмом при условии гладкости центральных слоев.

Так как многообразие $X_{0}$ горенштейново, то $\left|-K_{X}\right|$ вкладывает $X$ во взвешенное проективное пространство $\mathbb{P}_{\mathscr{O}}(1,1,2,3)$ над $\mathscr{O}$, при этом $X_{0}$ не проходит через особые точки $\mathbb{P}_{k}(1,1,2,3)$. Кроме того, хорошо известно, что собой представляет центральньй слой (см. $[14$, предложение 4.6] и $[15$, п. 1.4]):

ПРЕДЛОЖЕНИЕ 4.2. Если слой $X_{0}$ нормален, то либо все его особенности не Хуже чем дювалевские, либо существует единственная особая точка, являющаяся минимальной әллиптической.

Если слой $X_{0}$ ненормален, то его нормализачия есть $\mathbb{P}_{k}^{2} ;$ тогда $X_{0}$ получается "склейкой" плоскости вдоль квадрики (возможно, приводимой или неприведенной).

Отметим, что в любом из указанных случаев существует морфизм степени 2 на невырожденньй квадратичный конус в $\mathbb{P}_{k}^{3}$ с ветвлением в сечении кубикой, не проходящей через вершину конуса. Этот морфизм есть не что иное, как ограничение на $X_{0}$ проекции $\mathbb{P}_{k}(1,1,2,3) \rightarrow \mathbb{P}_{k}(1,1,2)$.

Следующее утверждение полезно для выделения горенштейновых канонических (в том числе терминальньх) особых точек [19].

ПРЕДЛОЖЕНИЕ 4.3. Пусть $U \rightarrow C$ - расслоение на поверхности дель Пецио над ростком гладкой кривой $C$, имеющее горенштейновые канонические особенности, $P$ - некоторая точка в чентральном слое. Тогда общий әлемент $\left|-K_{U}-P\right|$ имеет, самое большее, дювалевские особенности.

Наконец, для идентификации дювалевских особенностей будет применяться так назьваемьй “принцип распознавания" [20], которьй по уравнению (не обязательно в нормальной форме) позволяет определить, является ли данная особая точка дювалевской (и если да, то какой именно) или нет. Принцип заключается в следующем. Переменным в уравнении $f(x, y, z)=0$, задающем особую точку, приписывается всеми возможньми способами один из перечисленных ниже наборов весов:

$$
\left(\frac{1}{2}, \frac{1}{2}, \frac{1}{n+1}\right),\left(\frac{1}{2}, \frac{n-2}{2(n-1)}, \frac{1}{n-1}\right),\left(\frac{1}{2}, \frac{1}{3}, \frac{1}{4}\right), \quad\left(\frac{1}{2}, \frac{1}{3}, \frac{2}{9}\right), \quad\left(\frac{1}{2}, \frac{1}{3}, \frac{1}{5}\right) .
$$

Если для какого-нибудь из этих наборов минимальная взвешенная степень мономов равна 1 , то $f=0$ определяет дювалевскую особую точку типа $A_{n}, D_{n}, E_{6}, E_{7}$ или $E_{8}$ (соответственно перечисленным весам); в противном случае особенность не является дювалевской.

Дальнейшее изложение построено по следующему плану. Сначала мы предъявляем подходящую систему координат в $\mathbb{P}(1,1,2,3)$, в которой уравнения для $\varphi, X$ и $V$ записываются в удобной форме. Затем показьваем, что из получающегося при этом конечного числа возможных типов уравнений для $\varphi$ реализуется только один (работает условие горенштейновости), и приводим примеры перестроек. Последняя часть содержит ряд полезных замечаний о рассматриваемых перестройках. 
4.1. Подходящие координаты. Пусть м обозначает максимальньй идеал кольца $\mathscr{O}, t-$ его порождающий элемент, $\mathfrak{m}=(t) \mathscr{O}$.

Фиксируем экземпляр взвешенного проективного пространства $P_{1_{\mathscr{O}}} \cong \mathbb{P}_{\mathscr{O}}(1,1,2,3)$, в которое вложено $X$. Через $P_{1}$ и $P_{1_{K}}$ обозначим специализации $P_{1_{\mathscr{O}}}$ над центральной точкой $t=0$ и общей схемной точкой $R$ соответственно. Пусть $(x: y: z: w)$ координаты в $P_{1_{\mathscr{O}}}$ с весами $(1,1,2,3)$ (мы будем использовать те же обозначения координат для специализаций). Символы $P_{2}, P_{2}, P_{2_{K}}$ и $(p: q: r: s)$ зарезервированы для аналогичных объектов в случае $V$.

Многообразие $X$ определено (взвешенным) однородным полиномом степени 6 в $P_{1_{\mathscr{O}}}$ :

$$
a w^{2}+b z^{3}+c w z f_{1}(x, y)+z^{2} f_{2}(x, y)+z f_{4}(x, y)+f_{6}(x, y)=0
$$

где $a, b, c \in \mathscr{O}, f_{i}$ обозначает однородньй полином степени $i$. Так как $X_{0}$ не проходит через точки $(0: 0: 0: 1)$ и $(0: 0: 1: 0)$, то $a$ и $b$ обратимы в $\mathscr{O}$. В данном исследовании коэффициенты нас интересуют только “по модулю” $t$, так что можно просто положить всюду в дальнейшем $a=b=1$.

Заменив подходяшим образом координаты $w$ и $z$, можно упростить уравнение для $X$ :

$$
w^{2}+z^{3}+z f_{4}(x, y)+f_{6}(x, y)=0
$$

(здесь $f_{i}$ обозначают, очевидно, уже какие-то другие однородные полиномы). Легко проверить, что указанная замена координат индуцирует замену координат (а значит, автоморфизм) в центральном слое.

Далее, так как для $1 \leqslant j \leqslant 3$ вьполнены условия $H^{1}\left(P_{1_{K}}, \mathscr{I}_{X_{K}} \times \mathscr{O}(j)\right)=0$, где $\mathscr{I}_{X_{K}}$ - пучок идеалов $X_{K}$, мы видим, что

$$
H^{0}\left(X_{K},-j K_{X_{K}}\right) \simeq H^{0}\left(P_{1_{K}}, \mathscr{O}(j)\right) .
$$

Таким образом, изоморфизм $\varphi_{K}^{-1}: V_{K} \simeq X_{K}$ индуцирует изоморфизмы

$$
\left(\varphi_{K}^{-1}\right)^{*}: H^{0}\left(P_{1_{K}}, \mathscr{O}(j)\right) \simeq H^{0}\left(P_{2_{K}}, \mathscr{O}(j)\right),
$$

откуда следует, что $\varphi_{K}^{-1}$ расширяется до

$$
\varphi_{K}^{-1}: P_{2_{K}} \simeq P_{1_{K}}
$$

позволяя ввести следующие координаты на $P_{2_{K}}$ :

$$
\left\{\begin{array}{l}
p=\left(\varphi_{K}^{-1}\right)^{*}(x), \\
q=\left(\varphi_{K}^{-1}\right)^{*}(y), \\
r=\left(\varphi_{K}^{-1}\right)^{*}(z), \\
s=\left(\varphi_{K}^{-1}\right)^{*}(w) .
\end{array}\right.
$$

Учитывая указанное отождествление $P_{1_{K}}$ и $P_{2_{K}}$, а также отображение специализации

$$
P_{2_{K}}=P_{2_{\mathscr{O}}} \underset{\mathscr{O}}{\otimes} \operatorname{Spec} K \longrightarrow P_{2_{\mathscr{O}}}
$$

легко видеть, что после умножения на некоторьй элемент $\mathscr{O}$ мы можем положить для координат в $P_{2_{\mathscr{O}}} \quad p=A x, q=B y, r=C z$ и $s=D w$, где $A, B, C, D \in \mathscr{O}$. Ясно, что 
эти коэффициенты можно упростить, "растянув" координаты умножением на подходящие обратимые элементы, так что окончательно мы будем иметь следующее уравнение для $\varphi$ :

$$
\varphi=\left\{\begin{array}{l}
p=t^{a} x \\
q=t^{b} y \\
r=t^{c} z \\
s=t^{d} w
\end{array}\right\}
$$

причем набор $(a, b, c, d)$ содержит хотя бы один нуль. Теперь очевидно, что

$$
\varphi^{-1}=\left\{\begin{array}{c}
x=t^{\alpha} p \\
y=t^{\beta} q \\
z=t^{\gamma} r \\
w=t^{\delta} s
\end{array}\right\}
$$

с тем же самым условием на набор $(\alpha, \beta, \gamma, \delta)$. Более того, учитьвая веса координат, из условия $\varphi \circ \varphi^{-1}=\mathrm{id}$ мы находим, что

$$
6(a+\alpha)=6(b+\beta)=3(c+\gamma)=2(d+\delta) .
$$

Подставляя (4.3) в уравнение (4.1) и вспоминая, что $V_{0}$ не проходит через точки $(0$ : $0: 1: 0)$ и $(0: 0: 0: 1)$, получаем уравнение на $V$

$$
s^{2}+r^{3}+r g_{2}(p, q)+g_{6}(p, q)=0
$$

и условие $3 \gamma=2 \delta$, где $g_{i}$ - некоторые однородные полиномы степени $i$.

Итак, суммируя вьшесказанное, можно утверждать, что существует положительное целое $m$ такое, что вьполнены следующие условия:

$$
\left\{\begin{array}{l}
a+\alpha=m, \\
b+\beta=m \\
c+\gamma=2 m \\
d+\delta=3 m \\
2 d=3 c \\
2 \delta=3 \gamma
\end{array}\right.
$$

4.2. Разбор случаев. Используя симметрию ситуации, мы можем выделить четыре возможных случая:

\begin{tabular}{|c|c|c|c|}
\hline Случай & $(a, b, c, d)$ & $(\alpha, \beta, \gamma, \delta)$ & Замечания \\
\hline \hline $\mathrm{A}$ & $(a, m, 0,0)$ & $(\alpha, m, 2 m, 3 m)$ & $\begin{array}{c}a+\alpha=m \\
a, \alpha>0\end{array}$ \\
\hline $\mathrm{B}$ & $(0, m, 0,0)$ & $(m, 0,2 m, 3 m)$ & \\
\hline $\mathrm{C}$ & $(m, m, 0,0)$ & $(0,0,2 m, 3 m)$ & \\
\hline $\mathrm{D}$ & $(0, m, 2 k, 3 k)$ & $(m, 0,2 l, 3 l)$ & $k+l=m$ \\
& & & $k \geqslant 2$ \\
& & & $k, l>0$ \\
\hline
\end{tabular}


Мы сейчас покажем, что, на самом деле, первые три случая не реализуются.

Прежде всего, полагая, что поверхности $X$ и $V$ заданы уравнениями (4.1) и (4.4), а отображения $\varphi$ и $\varphi^{-1}$ - уравнениями (4.2) и (4.3), мы получаем следующие условия:

$$
\left\{\begin{array} { l } 
{ f _ { 4 } ( x , y ) = t ^ { c - 2 d } g _ { 4 } ( t ^ { a } x , t ^ { b } y ) , } \\
{ f _ { 6 } ( x , y ) = t ^ { - 2 d } g _ { 6 } ( t ^ { a } x , t ^ { b } y ) , }
\end{array} \quad \left\{\begin{array}{l}
g_{4}(p, q)=t^{\gamma-2 \delta} f_{4}\left(t^{\alpha} x, t^{\beta} y\right) \\
g_{6}(p, q)=t^{-2 \delta} f_{6}\left(t^{\alpha} x, t^{\beta} y\right)
\end{array}\right.\right.
$$

Будем считать, что $g_{4}$ и $g_{6}$ определены как

$$
g_{4}(p, q)=\sum_{i=0}^{4} a_{i} p^{4-i} q^{i}, \quad g_{6}(p, q)=\sum_{i=0}^{6} b_{i} p^{6-i} q^{i}
$$

Случай А. Здесь мы имеем

$$
\begin{aligned}
& f_{4}(x, y)=\sum_{i=0}^{4} a_{i} t^{4 a+(m-a) i} x^{4-i} y^{i}=t^{4 a} g_{4}\left(x, t^{m-a} y\right) \\
& f_{6}(x, y)=\sum_{i=0}^{6} b_{i} t^{6 a+(m-a) i} x^{6-i} y^{i}=t^{6 a} g_{6}\left(x, t^{m-a} y\right)
\end{aligned}
$$

Таким образом, многообразие $X$ определено уравнением

$$
w^{2}+z^{3}+t^{4 a} z g_{4}\left(x, t^{m-a} y\right)+t^{6 a} g_{6}\left(x, t^{m-a} y\right)=0
$$

Так как $a>0$, кривая $\{t=w=z=0\}$ лежит на $X$, причем $X$ сингулярно вдоль нее. Очевидно, такая особенность не может быть терминальной.

Случай С. Те же рассуждения: достаточно заменить $a$ на $m$.

Случай В. Мы имеем

$$
f_{4}(x, y)=\sum_{i=0}^{4} a_{i} t^{m i} x^{4-i} y^{i}, \quad f_{6}(x, y)=\sum_{i=0}^{6} b_{i} t^{m i} x^{6-i} y^{i}
$$

так что $X$ задано уравнением

$$
w^{2}+z^{3}+z\left(\sum_{i=0}^{4} a_{i} t^{m i} x^{4-i} y^{i}\right)+\sum_{i=0}^{6} b_{i} t^{m i} x^{6-i} y^{i}=0 .
$$

Заметим, что хотя бы один из коэффициентов этого уравнения не равен тождественно нулю. Выберем аффинную часть $\{y \neq 0\}$. Используя те же обозначения координат и для аффинной части (т.е. "по модулю” деления на $y$ ), мы видим, что точка $A=\{t=x=z=w=0\}$ особая на $X$.

Далее, уравнение общего элемента из $\left|-K_{X}\right|$, проходящего через эту точку, имеет вид $\{x=t h\}$ для некоторого $h \in \mathscr{O}$. Значит, такой элемент задан как

$$
w^{2}+z^{3}+u z t^{4}+v t^{6}=0
$$

где среди $u, v \in \mathscr{O}$ хотя бы один ненулевой. Согласно “принципу распознавания" такое уравнение не может задавать дювалевскую особенность (на самом деле, это уравнение 
минимальной эллиптической особенности). Таким образом, мы опять столкнулись с нетерминальным случаем (предложение 4.3).

4.3. Единственная возможность: случай D. Всюду далее будем полагать, что

$$
\varphi=\left\{\begin{array}{l}
p=x \\
q=t^{m} y \\
r=t^{2 k} z \\
s=t^{3 k} w
\end{array}\right\}, \quad \varphi^{-1}=\left\{\begin{array}{l}
x=t^{m} p \\
y=q \\
z=t^{2 l} r \\
w=t^{3 l} s
\end{array}\right\}
$$

где $k+l=m$ и $0<k \leqslant l$ (таким образом, $k \leqslant m / 2$ ).

Из (4.6) мы находим, что

$$
f_{4}(x, y)=t^{-4 k} g_{4}\left(x, t^{m} y\right), \quad f_{6}(x, y)=t^{-6 k} g_{6}\left(x, t^{m} y\right) .
$$

Значит, существуют $\alpha_{0}, \alpha_{1}, \beta_{0}, \beta_{1}, \beta_{2} \in \mathscr{O}$ такие, что

$$
\left\{\begin{array}{l}
a_{0}=\alpha_{0} t^{4 k} \\
a_{1}=\alpha_{1} t^{4 k-m} \\
b_{0}=\beta_{0} t^{6 k} \\
b_{1}=\beta_{1} t^{6 k-m} \\
b_{2}=\beta_{2} t^{6 k-2 m}
\end{array}\right.
$$

Дальнейшие рассуждения представляют собой “анализ многочленов" и, чтобы облегчить их восприятие, запишем нужные нам уравнения:

$$
\begin{aligned}
f_{4}(x, y)= & \alpha_{0} x^{4}+\alpha_{1} x^{3} y+a_{2} t^{2 m-4 k} x^{2} y^{2}+a_{3} t^{3 m-4 k} x y^{3}+a_{4} t^{4 m-4 k} y^{4}, \\
f_{6}(x, y)= & \beta_{0} x^{6}+\beta_{1} x^{5} y+\beta_{2} x^{4} y^{2}+b_{3} t^{3 m-6 k} x^{3} y^{3}+b_{4} t^{4 m-6 k} x^{2} y^{4} \\
& \quad+b_{5} t^{5 m-6 k} x y^{5}+b_{6} t^{6 m-6 k} y^{6}, \\
g_{4}(p, q)= & \alpha_{0} t^{4 k} p^{4}+\alpha_{1} t^{4 k-m} p^{3} q+a_{2} p^{2} q^{2}+a_{3} p q^{3}+a_{4} q^{4}, \\
g_{6}(p, q)= & \beta_{0} t^{6 k} p^{6}+\beta_{1} t^{6 k-m} p^{5} q+\beta_{2} t^{6 k-2 m} p^{4} q^{2}+b_{3} p^{3} q^{3}+b_{4} p^{2} q^{4}+b_{5} p q^{5}+b_{6} q^{6}
\end{aligned}
$$

И

$$
\begin{aligned}
X: w^{2} & +z^{3}+z\left(\alpha_{0} x^{4}+\alpha_{1} x^{3} y+t^{2 m-4 k} \sum_{i=2}^{4} a_{i} t^{m(i-2)} x^{4-i} y^{i}\right) \\
& +\beta_{0} x^{6}+\beta_{1} x^{5} y+\beta_{2} x^{4} y^{2}+t^{3 m-6 k} \sum_{i=3}^{6} b_{i} t^{m(i-3)} x^{6-i} y^{i}=0 \\
V: s^{2} & +r^{3}+r\left(\alpha_{0} t^{4 k} p^{4}+\alpha_{1} t^{4 k-m} p^{3} q+\sum_{i=2}^{4} a_{i} p^{4-i} q^{i}\right) \\
& +\beta_{0} t^{6 k} p^{6}+\beta_{1} t^{6 k-m} p^{5} q+\beta_{2} t^{6 k-2 m} p^{4} q^{2}+\sum_{i=3}^{6} b_{i} p^{6-i} q^{i}=0 .
\end{aligned}
$$

Заметим, что $\varphi$ не определено вдоль кривой $\{t=x=0\}$, а $\varphi^{-1}$-вдоль $\{t=q=0\}$. Далее, $V_{0}$ стягивается в точку $A=\{t=0,(0: 1: 0: 0)\}$, а $X_{0}-$ в $\{t=0,(1: 0: 0: 0)\}$. 
ПРЕДЛОЖЕНИЕ 4.4. Поверхность $X$ всегда имеет особенность.

ДокАЗАтЕльство. Так как $2 m-4 k \geqslant 0$, из (4.10) следует, что точка $A$ всегда особая на $X$. Предложение доказано.

Попутно мы доказали важный результат, усиливающий утверждение работы [18]:

СлЕДСТвИЕ 4.5. Пусть U/T - Мори-расслоение на поверхности дель Пеццо степени 1 над (проективной) кривой T. Класс $\mathfrak{M}_{b}(U / T)$ содержит не более одного неособого әлемента.

ЛЕмМА 4.6. Всегда выполняется неравенство $m \leqslant 6 k$.

ДокАЗАТЕЛЬСтво. Предположим противное, т.е. $m>6 k$. Тогда $\alpha_{1}, \beta_{1} \in \mathfrak{m}$ и $\beta_{2} \in \mathfrak{m}^{6}$ ввиду (4.8). Пусть $S=\{x=t h\}, h \in \mathscr{O},-$ общий элемент $\left|-K_{X}-A\right|$ (мы рассматриваем аффинную часть $\{y \neq 0\}$ ). Тогда $S$ имеет вид

$$
w^{2}+z^{3}+u z t^{4}+v t^{6}=0
$$

для некоторых $u, v \in \mathscr{O}$. Таким образом, $S$ неканоничен (по “принципу распознавания"), что противоречит предложению 4.3. Лемма доказана.

Рассмотрим два возможных случая: $k=l$, т.е. $m=2 k$, и $k<l$.

Случай $k=l$. Рассмотрим аффинную часть $\{p \neq 0\}$ на $V$. В ней $V$ определено уравнением

$$
s^{2}+r^{3}+r\left(\alpha_{0} t^{4 k}+q(\ldots \text { terms } \ldots)\right)+\beta_{0} t^{6 k}+\beta_{1} t^{4 k} q+q^{2}(\ldots \text { terms } \ldots)=0
$$

где через “...terms . . ." обозначена сумма мономов, вид которой не важен для дальнеших рассуждений. Мы видим, что точка $B \in V$ всегда особая. Таким образом, если $k=l$, перестройки слоя сушествуют только между сингулярными многообразиями.

$C$ лучай $k<l$. Здесь было бы интересным понять, при каких условиях $V$ неособо. Так как $X$ стягивается в точку $B$, разумно прежде всего проверить $V$ именно в этой точке. Выберем аффинную часть $\{p \neq 0\}$. Из уравнений (4.10) следует, что $\beta_{1} t^{6 k-m} \notin \mathfrak{m}$, т.е. $6 k-m \geqslant 0$. Тогда по лемме $4.6 m=6 k$ и, значит, $\beta_{1}$ обратим в $\mathscr{O}$.

Таким образом, если $V$ гладко, то $(a, b, c, d)=(0,6 k, 2 k, 3 k)$ и $(\alpha, \beta, \gamma, \delta)=(6 k, 0$, $10 k, 15 k)$. Отметим, что в этом случае $\alpha_{1} \in \mathfrak{m}^{2 k}$ и $\beta_{2} \in \mathfrak{m}^{6 k}$. Легко проверить, что $X_{0}$ имеет единственную особую точку типа $E_{8}$ (это точка $A$ ), так что $X$ имеет там $c E_{8}$-особенность.

4.4. Примеры. Приведем несколько примеров перестроек слоя с интересньци свойствами.

ПРимеР 4.7 (“гладкий случай"). Положим $(a, b, c, d)=(0,6,2,3)$ и $(\alpha, \beta, \gamma, \delta)=$ $(6,0,10,15) ;$ поверхности $X$ и $V$ определены уравнениями

$$
X: w^{2}+z^{3}+x^{5} y+t^{24} x y^{5}=0, \quad V: s^{2}+r^{3}+p^{5} q+p q^{5}=0 .
$$

Легко проверить, что $V$ неособо, $X$ имеет особую точку типа $c E_{8}$. 
ПРимеР 4.8 ("бирациональньй автоморфизм"). Рассмотрим следующий набор весов: $(a, b, c, d)=(2,0,2,3),(\alpha, \beta, \gamma, \delta)=(0,2,2,3)$; уравнения поверхностей

$$
X: w^{2}+z^{3}+t^{4} x^{5} y+x y^{5}=0, \quad V: s^{2}+r^{3}+p^{5} q+t^{4} p q^{5}=0 .
$$

Заметим, что $X$ и $V$ бирегулярно изоморфны: достаточно рассмотреть замену координат $w=s, z=r, x=q, y=p$. Таким образом, мы можем предполагать, что $\varphi \in \operatorname{Bir}(X)$ определяется следуюшим преобразованием в $P_{1_{K}}$ :

$$
x \mapsto t^{-1} y, \quad y \mapsto t x, \quad z \mapsto z, \quad w \mapsto w
$$

Очевидно, поверхность $X$ имеет $c E_{8}$-особенность в центральном слое.

ПРИмЕР 4.9 (“ненормальные слои"). Мы приведем два примера с одними и теми же весами $(a, b, c, d)=(2,0,2,3)$ и $(\alpha, \beta, \gamma, \delta)=(0,2,2,3)$ такие, что $X_{0}$ и $V_{0}$ ненормальны. Оба случая также будут являться бирациональными автоморфизмами.

Первый случай. Многообразия $X$ и $V$ задаются уравнениями

$$
X: w^{2}+z^{3}+t z x^{2} y^{2}+t x^{6}+t^{7} y^{6}=0, \quad V: s^{2}+r^{3}+t r p^{2} q^{2}+t^{7} p^{6}+t q^{6}=0
$$

слой $X_{0}$ ненормален, он задается уравнением $w^{2}+z^{3}=0$ ( “каспидальный" случай в [15, п. 1.4]); многообразие $X$ имеет $c E_{7}$-точку в центральном слое.

Второй случай. Многообразия $X$ и $V$ задаются уравнениями

$X: w^{2}+z^{3}-3 z x^{2} y^{2}+t x^{6}+2 x^{3} y^{3}+t^{7} y^{6}=0, \quad V: s^{2}+r^{3}-3 r p^{2} q^{2}+t^{7} p^{6}+2 p^{3} q^{3}+t q^{6}=0 ;$

слой $X_{0}$ задан как $w^{2}+(z-x y)^{2}(z+2 x y)$ ("нодальньй" случай в $[15$, п. 1.4$\left.]\right)$; особая точка $X$ имеет тип $c D_{4}$.

4.5. Центральные слои. Напомним, что отображения $\varphi$ и $\varphi^{-1}$ стяпиают центральные слои в точки. Так как $X$ и $V$ предполагаются терминальными, то $X_{0}$ и $V_{0}$ должны быть рациональны. Если $X_{0}$ ненормален, то его нормализация есть $\mathbb{P}^{2}$ (предложение 4.2 ), т.е. $X_{0}$ рационален, как и следовало ожидать. Предположим, что $X_{0}$ нормален. Тогда он рационален, если и только если все его особенности не хуже, чем дювалевские. Таким образом, случай минимальной эллиптической особенности (задаваемой, например, уравнением $\left.w^{2}+z\left(z^{2}-x^{4}\right)=0\right)$ в центральном слое невозможен.

4.6. Антиканонические дивизоры. Пусть $S \in\left|-K_{X}\right|-$ общий элемент, $S^{\prime} \in\left|-K_{V}\right|-$ его собственньй прообраз при перестройке слоя. Очевидно, $S$ - гладкая эллиптическая поверхность. Покажем, что эллиптическая поверхность $S^{\prime}$ имеет эллиптическую (не обязательно минимальную) особенность в центральном слое.

Обозначим через $s$ и $s^{\prime}$ сечения $S$ и $S^{\prime}$, которые являются базисньми кривыми антиканонических линейных систем на $X$ и $V$. Пусть $\rho: T \rightarrow S^{\prime}$ - минимальное разрешение особенностей $S^{\prime}$. Так как $S$ относительно минимальна над $R$, мы имеем бирациональный морфизм $\mu: T \rightarrow S$, которьй, очевидно, может быть разложен в композицию стягиваний -1-кривых. Пусть $C$ и $C^{\prime}$ - центральные слои $S$ и $S^{\prime}, \tilde{C}$ и $\tilde{C}^{\prime}$ - их собственныепрообразы на $T$, а $s_{T}$ - прообраз $s$ (или $s^{\prime}$ ) там же. Очевидно, $C$ есть либо эллиптическая кривая, либо рациональная кривая с двойной точкой (каспидальной или нодальной). Мы знаем, что отображение $\rho$ стягивает $\tilde{C}$ в точку на $S^{\prime}$, а отображение $\mu$ стягивает $\tilde{C}^{\prime}$. Так как $C^{\prime}$ неприводима, $\tilde{C}^{\prime}$ - единственная - 1-кривая в центральном слое $T$. Более того, 


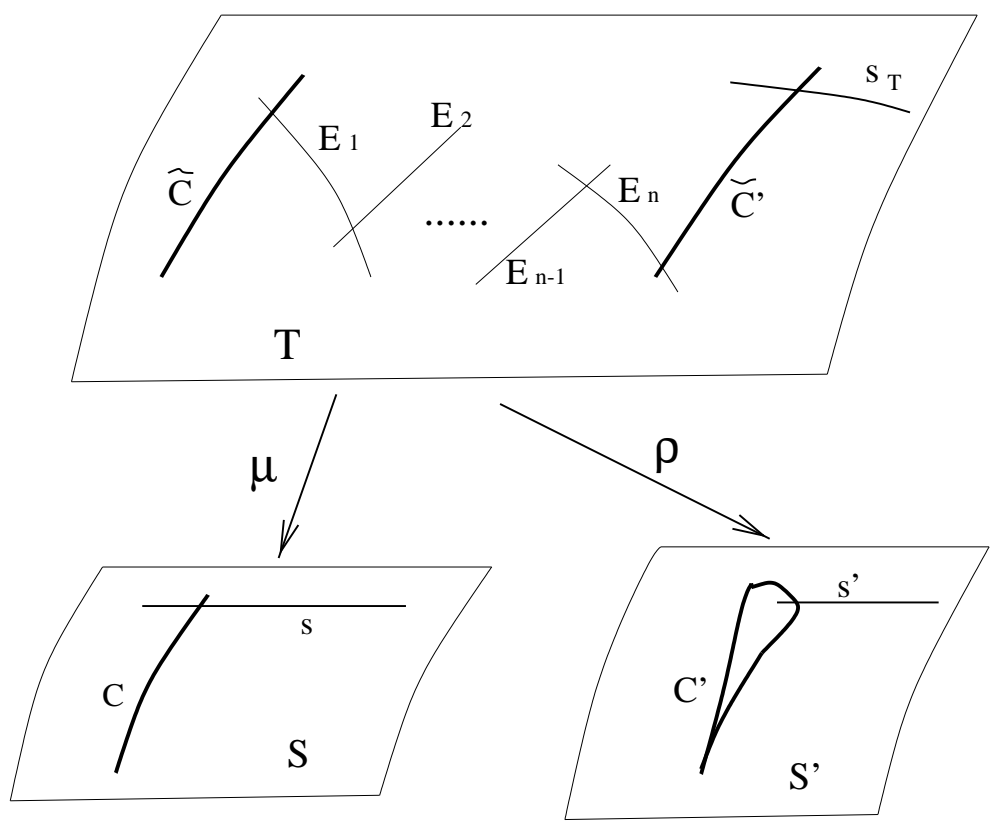

Рис. 1

$s^{\prime} \cap C^{\prime}$ - неособая точка на $S^{\prime}$, поэтому $s_{T}$ не пересекает исключительные кривые разрешения $\rho$. Далее, точка $s \cap C$ неособа для $C$, так что $\tilde{C}$ имеет арифметический род 1 . Рисунок 1 проясняет геометрическую ситуацию. На нем $E_{1}, \ldots, E_{n}, n \geqslant 0$, обозначают -2-кривые. Отметим, что $C^{\prime}$ обязательно имеет каспидальную особенность.

Таким образом, мы имеем следующие индексы пересечений: $\tilde{C}^{2}=\tilde{C}^{\prime 2}=-1, E_{i}^{2}=-2$, $E_{i} \circ E_{i+1}=1$.

Далее, морфизмы $\rho$ и $\mu$ позволяют записать $K_{T}$ двояким образом:

$$
K_{T} \sim-(n+1) \tilde{C}-n E_{1}-(n-1) E_{2}-\cdots-E_{n}
$$

и

$$
K_{T} \sim E_{1}+2 E_{2}+\cdots+n E_{n}+(n+1) \tilde{C}^{\prime} .
$$

Сравнивая эти два выражения, мы видим, что

$$
(n+1)\left(\tilde{C}+E_{1}+\cdots+E_{n}+\tilde{C}^{\prime}\right) \sim 0 .
$$

Смысл этой формулы (заметим, что $\tilde{C}+E_{1}+\cdots+E_{n}+\tilde{C}^{\prime}-$ слой $T$ ) можно пояснить так. Предположим, что $S$ и $S^{\prime}$ проективны (другими словами, база $R$ - проективная кривая), $f$ и $f^{\prime}$ - классы слоев этих поверхностей. Существуют целые $m$ и $m^{\prime}$ такие, что $K_{S} \sim m f$ и $K_{S^{\prime}} \sim m^{\prime} f^{\prime}$. Тогда

$$
m^{\prime}=m+(n+1) .
$$

Очевидно, $S^{\prime}$ имеет эллиптическую особенность, при этом ее фундаментальньй цикл есть $\tilde{C}$. Минимальной эллиптической эта особенность будет, если и только если $n=0$. 


\section{СПИСОК ЦИТИРОВАННОЙ ЛИТЕРАТУРЫ}

[1] Исковских В. А. Бирациональные автоморфизмы трехмерных алгебраических многообразий // Итоги науки и техники. Совр. пробл. матем. Т. 12. М.: ВИНИТИ, 1979. С. 159-236.

[2] Пухликов А.В. Бирациональные автоморфизмы трехмерных алгебраических многообразий с пучком поверхностей дель Пеццо // Изв. РАН. Сер. матем. 1998. Т. 62. № 1. С. 123-164.

[3] Саркисов В. Г. Бирациональные автоморфизмы расслоений на коники // Изв. РАН. Сер. матем. 1980. Т. 44. № 4. С. 918-944.

[4] Corti A. Singularities of linear systems and 3-fold birational geometry. Warwick Preprint 11/1999. Warwick, 1999.

[5] Kollar J., Mori S. Birational Geometry of Algebraic Varieties. With the Collaboration of C. H. Clemens and A. Corti. Cambridge Tracts in Math. V. 134. Cambridge: Cambridge Univ. Press, 1998.

[6] Исковских В. А., Манин Ю. И. Трехмерные квартики и контрпримеры к проблеме Люрота // Матем. сб. 1971. Т. 86(128). №1. С. 140-166.

[7] Гриненко М. М. Бирациональные свойства пучков поверхностей дель Пеццо степеней 1 и 2 // Матем. сб. 2000. Т. 191. №5. С. 17-38.

[8] Гриненко М. М. Бирациональные автоморфизмы трехмерного двойного конуса // Матем. сб. 1998. Т. 189. № 7. С. 37-52.

[9] Исковских В.А. Факторизация бирациональных отображений рациональных поверхностей с точки зрения теории Мори // УМН. 1996. Т. 51. № 4. С. 3-72.

[10] Манин Ю. И. Рациональные поверхности над совершенными полями // Publ. Math. IHÉS. 1996. V. 30. P. 55-97.

[11] Kollar J. (ed. ) Flips and Abundance for Algebraic Threefolds. A Summer Seminar at the University of Utah (Salt Lake City, 1991). Asterisque. V. 211. Paris: Soc. Math. de France, 1992.

[12] Corti A. Factoring birational maps of threefolds after Sarkisov // J. Algebraic Geom. 1995. V. 4. P. 223-254.

[13] Bruno A., Matsuki K. Log Sarkisov program // International J. Math. 1997. V. 8. № 4. P. 451-494.

[14] Hidaka F., Watanabe K.-i. Normal Gorenstein surfaces with ample anti-canonical divisor // Tokyo J. Math. 1981. V. 4. № 2. P. 319-330.

[15] Reid M. Nonnormal del Pezzo surfaces // Publ. Res. Inst. Math. Sci. 1994. V. 30. № 5. P. $695-727$.

[16] Исковских В.А.О проблеме рациональнсти для трехмерных алгебраических многообразий, расслоенных на поверхности дель Пеццо // Труды МИАН. 1995. Т. 208. С. 128-138.

[17] Corti A. Del Pezzo surfaces over Dedekind schemes // Ann. Math. (2). 1996. V. 144. № 3. P. 641-683.

[18] Park J. Birational maps of del Pezzo fibrations // E-print math/9912076, 1999.

[19] Кудрявцев С. А. О существовании хорошего дивизора для расслоения на поверхности дель Пеццо // Вестн. МГУ. Сер. 1. Матем., мех. 1998. № 2. С. 38-42.

[20] Bruce J. W., Wall C. T. C. On the classification of cubic surfaces // J. London Math. Soc. (2). 1979. V. 19. P. 245-256. 